\begin{tabular}{|r|r|r|r|r|}
\hline Cuadernos de Investigación Geográfica & 2003 & N$^{\circ} 29$ & pp. 35-49 & ISSN 0211-6820 \\
\hline
\end{tabular}

(C) Universidad de La Rioja

\title{
LAS FORMACIONES VEGETALES COLONIZADORAS DE LOS ESPACIOS QUEMADOS PREPIRENAICOS
}

\author{
F. PÉREZ CABELLO(*) \\ P. IBARRA BENLLOCH
}

Departamento de Geografía y Ordenación del Territorio (Universidad de Zaragoza).

Ciudad Universitaria s/n. Zaragoza 50.009.

(*)Lavasier@posta.unizar.es

RESUMEN: Se analiza la respuesta fisionómica y florística de una serie de comunidades vegetales representativas del Prepirineo oscense, afectadas por incendios forestales a mediados de la década de los ochenta. Teniendo en cuenta la superficie ocupada por estratos y el recubrimiento de las especies más representativas de cada comunidad, se realizan análisis comparativos entre las formaciones vegetales postincendio y parcelas control no afectadas. Los resultados finales muestran la enorme resilencia de las comunidades analizadas, lo que coincide con la mayor parte de los estudios recientes sobre regeneración en zonas quemadas del ámbito mediterráneo. Sólo en el caso de las formaciones que sustituyen a las comunidades de Pinus sylvestris se han detectado diferencias significativas en relación con las comunidades no afectadas. No obstante, las formaciones que aparecen en los espacios quemados presentan un importante incremento de la cubierta de matorral (acusado incremento de Genista scorpius) y una mayor presencia de especies herbáceas competitivas.

ABSTRACT: In this paper, structure and floristic response of representative Western Pre-Pyrenees communities that have been burned in the mid-eighties are analyzed. Different comparisons are made between postfire vegetal formations and unburned control plots, considering both the area occupied by stratum and the cover of the most representative species in each community. As most of the recent studies in the Mediterranean area do, final results show the great resilience of the analyzed communities, which agrees with most of the recent studies in the Mediterranean area. Significant fisionomic-floristic variations have been detected only in the case of formations that replace Pinus sylvestris communities. However, communities appearing in burned areas present an important increase in the brushland cover (a clear dominance of Genista scorpius) and a greater presence of competitive herbaceous species.

Palabra Clave: Prepirineo occidental, comunidades vegetales mediterráneas, incendio forestal, regeneración postincendio.

Key words: Western pre-Pyrenees, Mediterranean vegetal communities, forest fire, post-fire regeneration. 


\section{Introducción y objetivos}

En el marco del Pirineo aragonés, el Prepirineo occidental oscense es uno de los sectores más seriamente afectados por las consecuencias negativas de los incendios forestales -desorganización de la estructura vegetal, alteración de los ciclos hidrológicos,... -. No en vano, en el último cuarto del siglo XX (1974-1999) en este área se han calcinado cerca de 24.000 has, que representan el 50\% de la superficie afectada en la provincia de Huesca y el 14,12\% del total de Aragón (Pérez-Cabello, 2002).

A pesar de los efectos negativos del fuego, especialmente en lo que a la vegetación se refiere, la eficacia y profusión de los mecanismos de rebrotación-germinación de la mayoría de las especies vegetales que integran las comunidades submediterráneas prepirenaicas deriva, en muchas ocasiones, en un rápido proceso de recubrimiento vegetal, presentando caracteres similares a los de las comunidades anteriores. A este proceso de regeneración, aceptado en términos generales en los ecosistemas mediterráneos (Papió, 1988; May, 1991), se le denomina en la bibliografía autosucesión (Hanes, 1971). En los trabajos de Naveh (1990); Trabaud (1990, 1998, 2002); Tárrega y Luis-Calabuig (1987, 1989, 1990); Vera de la Fuente (1994); Badía et al., (1995) se describe este fenómeno, concluyéndose que la regeneración posterior al fuego en estos medios se asemeja a un proceso de autosucesión compensatorio a la regresión sufrida.

Sea como fuere, de lo que no cabe duda es de la especificidad del proceso de sucesión vegetal debido a la parcial destrucción del contenido biológico -supervivencia de la biomasa subterránea y de los bancos de semillas- como consecuencia de la mala conductividad térmica del suelo (Tárrega y Luis-Calabuig, 1989), y de la conservación de ciertas condiciones edáficas.

En este contexto, el objetivo de este trabajo es describir, en relación con una serie de incendios ocurridos entre 1985 y 1986, las características fisionómicas y las nuevas relaciones de dominancia que se establecen entre los principales taxones de una serie de comunidades, haciendo hincapié en la identificación de patrones comunes de respuesta y en el análisis de sus relaciones con la vegetación preexistente. De este modo, a partir de una aproximación fitocenósica, se puede diferenciar la rapidez de cicatrización (Gordon y Poissonet, 1973) de las diferentes comunidades, en términos de grado de difordonpara volver a su estado anterior al impacto en condiciones naturales.

La hipótesis de partida que preside el trabajo se centra en que, debido al carácter mediterráneo de la mayor parte de las comunidades prepirenaicas y al hecho de que muchas de ellas son sistemas preadaptados al fuego en las que dominan las especies retoñadoras, la mayoría de las formaciones que colonizan las zonas quemadas guardan una estrecha relación con las de partida, de modo que las características de estas últimas constituyen el factor con mayor capacidad explicativa del proceso de restauración vegetal.

Sobre la base del grado de siniestralidad en el intervalo temporal escogido, se ha analizado el proceso de reconstrucción en quejigares, encinares, pinares de silvestre, pinares de carrasco y formaciones arbustivas de boj, todas ellas comunidades representativas de la vegetación prepirenaica. 
Los quejigares se incluyen dentro de la alianza Buxo-Quercenion pubescenti-petraeae y representan la vegetación climácica del ámbito prepirenaico. Los carrascales seleccionados ocupan los ambientes xerofíticos del piso montano, constituyendo una variante de ambientes secos de la asociación Buxo-Quercetum rotundifoliae (Quercetum rotunfiloliae subas. Buxetosum). Los pinares albares se incluyen en la subasociación BuxoQuercetum pubescentis pinetosum pyrenaicae de ambientes montanos continentalizados, mientras que los presididos por Pinus halepensis aparecen habitualmente acompañados de Juniperus oxycedrus, Quercus coccifera y Genista scorpius, y representan la comunidad principal que tapiza los sectores más cálidos y secos de la Depresión de La Peña. Las fruticedas de boj, se corresponden con diferentes etapas de degradación de las comunidades de quercíneas subesclerófilas y coníferas de la zona de estudio que pueden insertarse en la alianza Amelanchiero-Buxenion cuando aparecen dominadas por Buxus sempervirens y otros especies arbustivas submediterráneas xerofíticas.

\section{Presentación de las áreas test y metodología aplicada}

Las áreas test analizadas se sitúan en superficies calcinadas entre 1985 y 1986. La selección de esta ventana en el tiempo responde a la necesidad de comparar espacios con los mismos antecedentes en relación con el momento de la última perturbación. Los incendios donde se han situado las áreas test se recogen a continuación (Figura 1). Los números entre paréntesis que acompañan a la denominación general identifican el parte de incendio y el año en el que tuvo lugar el fuego, respectivamente:

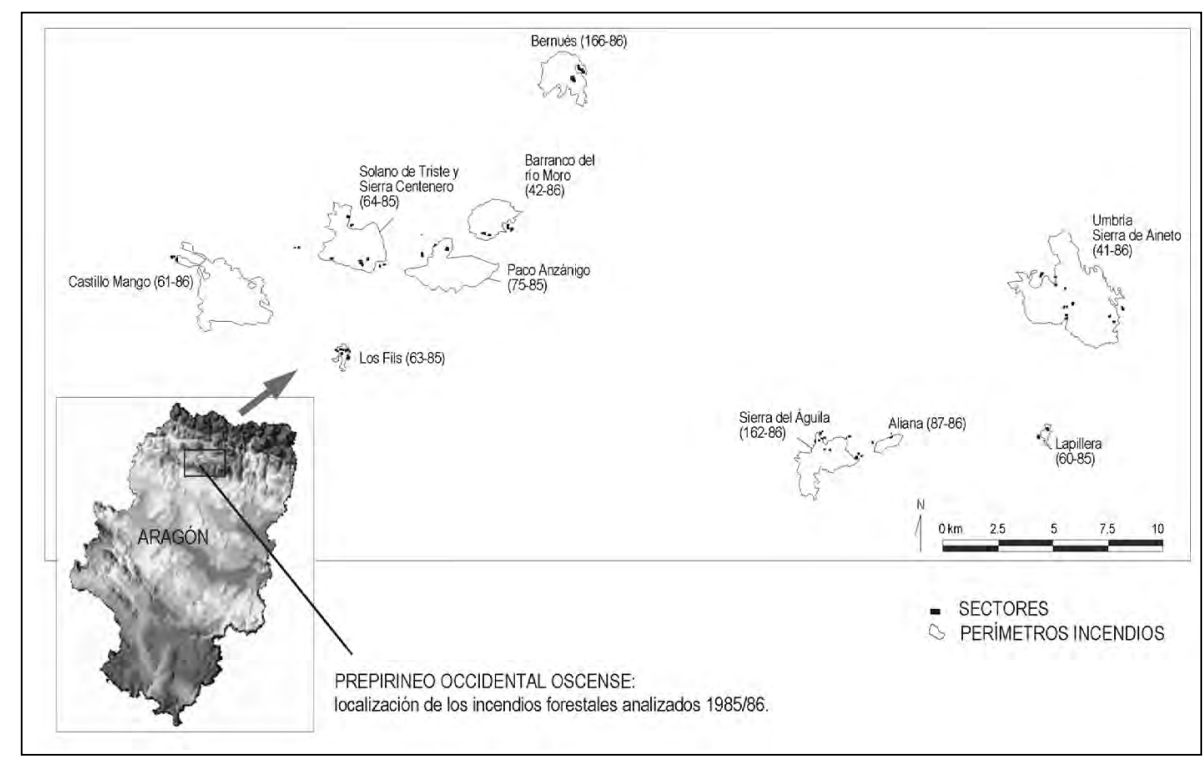

Figura 1. Localización del área de estudio y distribución de las áreas test.

- Incendio del Solano de Triste y Sierra de Centenero (64-85), término municipal de Las Peñas de Riglos. Margen derecha del Gállego en una ladera con exposición sureste, entre la Sierra de Centenero al norte y la Sierra de Sta. Isabel al oeste. 
- Incendio de "Castillo Mango" (61-86), término municipal de Agüero. Paraje llamado "Castillo Mango", entre las cabeceras de los Bco. de Los Felices y Rebosera.

- Incendio del Paco Anzánigo (75-85), términos municipales de Las Peñas de Riglos

y Caldearenas. Ladera norte, en la margen izquierda del río Gállego al este de Anzánigo.

- Incendio del Barranco del río Moro (42-86), término municipal de Las Peñas de Riglos-Caldearenas. Margen izquierda del Gállego a su paso por Anzánigo.

Incendio de la Umbría de la Sierra de Aineto (41-86), término municipal de Sabiñánigo. Margen izquierda del río Guarga, al sur de San Estebán y Lasaosa.

- Incendio de la Sierra del Águila (162-86), término municipal de Nueno. Cabeceras de los barrancos de Sabuco, Collado, Escalepones y Terrao situados en los flancos oriental y occidental del Pico del Águila.

- Incendio de Bernués (166-86), término municipal de Jaca. Al norte de la localidad de Bernués.

- Incendio de Paco Aliana (87-86), término municipal de Nueno. Vertiente norte de los acantilados de Cienfuens.

- Incendio de Lapillera (60-85), término municipal de Nueno. Base de la Punta de Corcurezo, al sur de la localidad de Nocito.

- Incendio de Los Fils (63-85), término municipal de Las Peñas de Riglos. Sureste de los Mallos de Riglos.

Cada una de las áreas test seleccionadas está formada por un sector quemado (localizado dentro del perímetro del incendio) y un sector no quemado o control, con las mismas características físicas que el anterior pero sin incendiar. Estas réplicas -sectores control- habitualmente se localizan en zonas próximas al perímetro exterior de las áreas incendiadas o en pequeñas isletas dentro de los contornos que no han sido afectadas por el fuego. En cuanto a los sectores quemados se procura seleccionar áreas que hayan sufrido niveles de intensidad de fuego similar, $y$, evidentemente que no se hayan visto modificadas por actuaciones de origen antrópico -repoblaciones, pastoreo, etc.-. En total se han prospectado 39 áreas test: 9 correspondientes a zonas de quejigar, 8 a encinar, 7 a pinar de silvestre, 7 a pinar de carrasco y 8 a fruticedas dominadas por el boj.

El análisis de la vegetación se basa en la realización, durante el verano de 1999, de 78 inventarios florísticos siguiendo la metodología de Braun-Blanquet (1979), incorporando el análisis por estratos de Bertrand (1966). No obstante, se estima el porcentaje de recubrimiento de cada especie en relación con la superficie del inventario y con la del estrato donde se sitúa. Por tanto, los índices de abundancia/dominancia se aplican a cada 
uno de los estratos conformantes y a las especies vegetales integradas en cada uno de ellos tomando como referencia las propias dimensiones del estrato donde se inscriben.

En atención a los objetivos marcados, la metodología aplicada se estructura en dos etapas diferentes:

En la primera se acometen sendos análisis descriptivos de la respuesta vegetal diferenciando por estratos y comunidades, calibrando el grado de recuperación de las comunidades afectadas en relación con dos variables sintéticas: el índice de variación en las relaciones de dominancia interespecie (IVD), y el índice de variación fisionómica (IVF). El primer índice computa, en cada comunidad, las diferencias entre los sectores control y quemado en relación con el porcentaje de ocupación de las principales especies representativas. El segundo registra las diferencias entre los sectores control y quemado en relación con el porcentaje de recubrimiento de los diferentes estratos. Matemáticamente los índices representan los promedios de los residuales calculados como las diferencias absolutas entre los valores de los sectores control y quemados, todo ello partido por 100, de tal modo que los valores resultantes quedan comprendidos en un rango entre 1 y 0 . La expresión matemática se presenta a continuación:

$$
\begin{aligned}
& \alpha=\frac{\sum_{n=1}^{5}\left({ }_{E q_{n}-E t_{n}}\right) * 100}{n} \\
& I V F=\operatorname{prom} \sum_{n=1}^{n} \alpha
\end{aligned}
$$

$$
\begin{gathered}
\alpha=\frac{\sum_{n=1}^{5}\left({ }_{q_{n}-T_{t}}\right) * 100}{n} \\
I V D=\operatorname{prom} \sum_{n=1}^{n} \alpha
\end{gathered}
$$

Donde $E q_{n}$ y $E t_{n}$ son los porcentajes de recubrimiento por estrato de los sectores quemados y control, respectivamente, y $T q_{n}$ y $T t_{n}$ los porcentajes de recubrimiento de la especie $T$ en los sectores quemados y control, respectivamente.

Los valores próximos a " 0 " expresan estructuras o relaciones de dominancia interespecies similares a los del sector control, mientras que los valores extremos "1" representan formaciones muy diferentes en relación con aquellas de las que proceden. Además, mediante la aplicación de contrastes sobre la igualdad de medias, se comparan los índices en las comunidades, dos a dos, con el propósito de detectar diferencias estadísticamente significativas entre sí.

En la segunda etapa se ensaya, mediante un análisis cluster, una taxonomía de respuestas frente al fuego y su correspondencia con las formaciones precedentes. En dicho análisis se consideran las variables más significativas que exhiban una gran diferencia entre grupos: los porcentajes de recubrimiento por estrato; el recubrimiento de las principales especies definitorias de las áreas test -quejigo, encina, pino carrasco, pino laricio, pino silvestre y boj-; y las especies con mayor predominio espacial -coscoja, enebro, rosas, romero, tomillo-. La elección de número de cluster viene determinado por la estructura previa de observaciones: cinco conglomerados, los correspondientes a cada tipo de comunidad vegetal. Se sigue un procedimiento jerárquico mediante métodos de 
aglomeración. En concreto se utiliza el método Ward por el cual la distancia entre dos conglomerados es la suma de los cuadrados entre dos conglomerados sumados para todas las variables. El sesgo derivado por las diferencias en las mediciones de los atributos analizados se ha eliminado mediante una función de estandarización. Todo el conjunto de operaciones estadísticas se ha realizado con el uso de las opciones ofrecidas por el paquete estadístico Statgraphics Plus for Windows 3.0 (Statistical Graphics Corp. 19941997).

\section{Resultados}

\subsection{Análisis descriptivo por estratos}

En términos generales, las herbáceas y las leñosas inferiores a $0.5 \mathrm{~m}$ ocupan el $57.77 \%$ de la superficie inventariada en las zonas quemadas -el $50.9 \%$ en los sectores control-. Constituye, por tanto, el estrato con mayor desarrollo superficial de los prospectados y el que presenta una mayor diversidad florística -162 especies diferentes-. En este conjunto, son dominantes caméfitos y nanofanerófitos como Buxus sempervirens y Genista scorpius y un gran número de herbáceas perennes, entre las cuales destacan algunos hemicriptófitos como Brachypodium ramosum, Aphyllanthes monspeliensis, etc.

En el estrato subarbustivo el porcentaje de ocupación representa el 31.5\% de la superficie del inventario $-23.8 \%$ en los sectores control-, sumando un total de 36 especies diferentes, pertenecientes, en su mayor parte, a las familias de las leguminosas, rosáceas y buxáceas. Dos de ellas presentan porcentajes de ocupación superiores al 30\%: Buxus sempervirens y Genista scorpius.

Además del incremento de la superficie de ocupación de este estrato en relación con los sectores control, los espacios quemados a este nivel también presentan importantes variaciones en las relaciones de dominancia interespecies. En este sentido destaca, por una lado, el leve incremento de algunas rosáceas -Rubus ulmifolius y Rosa canina- y del romero, Rosmarinus officinalis, y el espectacular crecimiento de Genista scorpius que pasa a ocupar un $18,71 \%$ más de extensión. Por otro lado, llama la atención, el descenso experimentado por Buxus sempervirens (19.64\%).

En el estrato arbustivo se ha reconocido un número de especies ligeramente inferior al estrato subarbustivo y evidentemente inferior al ESTRATO 128 especies). Ocupa el $32.2 \%$ del área inventariable $-34.6 \%$ en los sectores control-, por tanto, en términos espaciales es sensiblemente menos importante que los anteriores. Entre las especies que controlan este nivel, junto a los habituales caméfitos -Genista scorpius, Quercus coccifera, Buxus sempervirens- y algunos fanerófitos pertenecientes a las rosáceas como Rubus ulmifolius, Rosa canina, Rosa arvensis, hay que destacar la presencia de Pinus halepensis y de los macrofanerófitos representativos del área de estudio (Quercus gr. cerrioides y Quercus rotundifoliae). Las relaciones de dominancia entre especies están más equilibradas, aunque sigue siendo el boj con el $28.24 \%$ la especie más representativa. No obstante, las pináceas y las rosáceas presentan niveles entre el 1 y el 4\%, y la encina, el quejigo, la coscoja y la aliaga presentan valores comprendidos entre el 11 y el $16 \%$. 
Un rasgo destacable en este estrato es la mayor representación espacial que tienen las especies arbóreas en los sectores quemados en relación con los que no lo están. La razón de este extremo se relaciona con que, tras catorce años después del fuego, una parte importante de la vegetación arbórea afectada por el fuego ha sido capaz de posicionar individuos en este estrato gracias a sus sistemas de regeneración; en el caso del quejigo y de la encina mediante multiplicaciones vegetativas y en el caso del pino carrasco mediante germinación.

En lo referente a los estratos superiores -ESTRATOS ${ }_{4 / 5-}$, aunque en las zonas quemadas puntualmente se han observado algunos pies aislados de Quercus gr. cerrioides con una talla cercana a los siete metros, el estrato arborescente marca el techo de la vegetación regenerada tras los fuegos de mediados de los 80. En este contexto, el ESTRATO 4 cubre el $5,7 \%$ de la zona inventariada. Las especies registradas en este nivel se reducen considerablemente en relación con los estratos inferiores, recogiéndose tan solo siete taxones: Pinus nigra, Amelanchier ovalis, Rosa arvensis, Quercus rotundifoliae, Pinus halepensis y Quercus gr. cerrioides; con excepción del pino laricio, todas las demás presentan valores superiores al $1 \%$. En este sentido destaca la significativa presencia del pino carrasco $(25,39 \%)$ y especialmente la del quejigo, con el $60,41 \%$.

\subsection{Diagnóstico por comunidades vegetales}

En las Tablas 1, 2 y 3 y en la Figura 2 se presentan los resultados más significativos. En lo que respecta a los quejigares, las formaciones que los sustituyen presentan un recubrimiento acumulado cercano al $160 \%$, con una ocupación media por estrato del $31.4 \%$, lo que supone una disminución en 6 puntos en relación con los sectores control (37\%). Los estratos arbustivo, subarbustivo y, en especial, el estrato $1(>60 \%)$ definen la estructura de unas formaciones en las que los estratos superiores también están representados, si bien, de manera puntual en el caso del arbóreo (1.7\%) y más significativamente en el arborescente (16.1\%).

En términos relativos, el subarbustivo es el estrato que mayor desarrollo ha experimentado en relación con los sectores control (162.5\%); mientras que el arborescente, que pasa a ocupar la mitad de la superficie (52.7\%), y el arbóreo con una representación tan sólo del 4\% son los menos desarrollados. A pesar de este proceso de dilatación por la base en las estructuras de las formaciones postincendio, el índice de variación fisionómica (IVF) obtenido es de 0.27 lo cual, teniendo en cuenta el carácter arbóreo-arborescente de las comunidades pre-incendio y el relativamente corto periodo de tiempo que ha transcurrido desde la perturbación, se interpreta como un nivel de cicatrización bastante significativo, vaticinando una pronta recuperación de estas comunidades a medio plazo.

Las relaciones de dominancia en las formaciones post-incendio acompañan los resultados a nivel fisionómico. De este modo, la vegetación continúa estando presidida por Quercus gr. cerrioides y Buxus sempervirens, aunque en proporciones más reducidas que en los sectores control (23\% y 15\%, respectivamente). En el estrato subarbustivo destaca la abundancia de Buxus sempervirens y Genista scorpius, mientras que en el arbustivo a las anteriores hay que añadir Quercus gr. cerrioides y algunos fanerófitos como Amelanchier ovalis y Crataegus monogyna. 


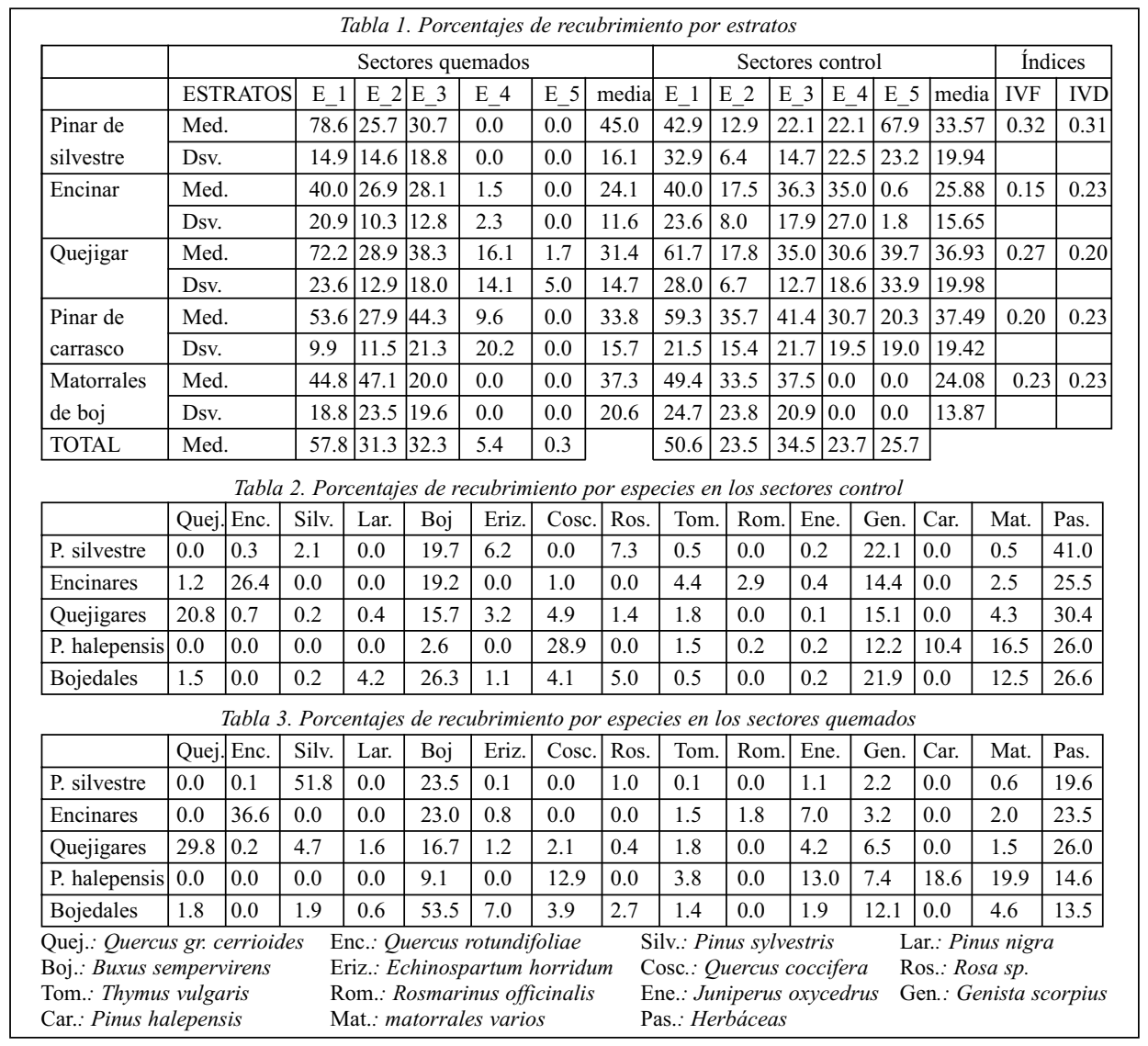

En términos relativos, las herbáceas continúan presentado un elevado nivel de ocupación (30.4\%) experimentando un leve incremento en relación con los sectores control (4 puntos). Genista scorpius protagoniza un incremento de casi 9 puntos lo que supone casi triplicar el espacio que ocupaba en los sectores control. Sin duda, este último dato evidencia un cierta degradación en la comunidad, al tiempo que pone de manifiesto la gran capacidad colonizadora de esta especie.

A pesar de la invasión de esta leguminosa, la buena recuperación de las especies representativas, Quercus gr. cerrioides (69.8\%) y Buxus sempervirens (94\%), mantienen, aunque parcialmente, las relaciones de dominancia, haciendo que el IVD sea relativamente bajo (0.20). En gran medida los buenos resultados de la recuperación de estas especies están relacionados con el desarrollo, durante los primeros años, de numerosos rebrotes y hojas lo que les permite un posicionamiento privilegiado desde el punto de vista de las relaciones de dominancia, sin duda, uno de los principales procesos controladores de la estructura y composición florística de estas comunidades.

Las formaciones que sustituyen al encinar se organizan en torno al ESTRATO ${ }_{1}$, los estratos subarbustivo y arbustivo -recubrimientos próximos al 30\%- y la presencia testi- 
Figura 2. Comparación de los porcentajes de recubrimientos por estrato entre los sectores control (izquierda.) y quemados (derecha.) por formaciones vegetales
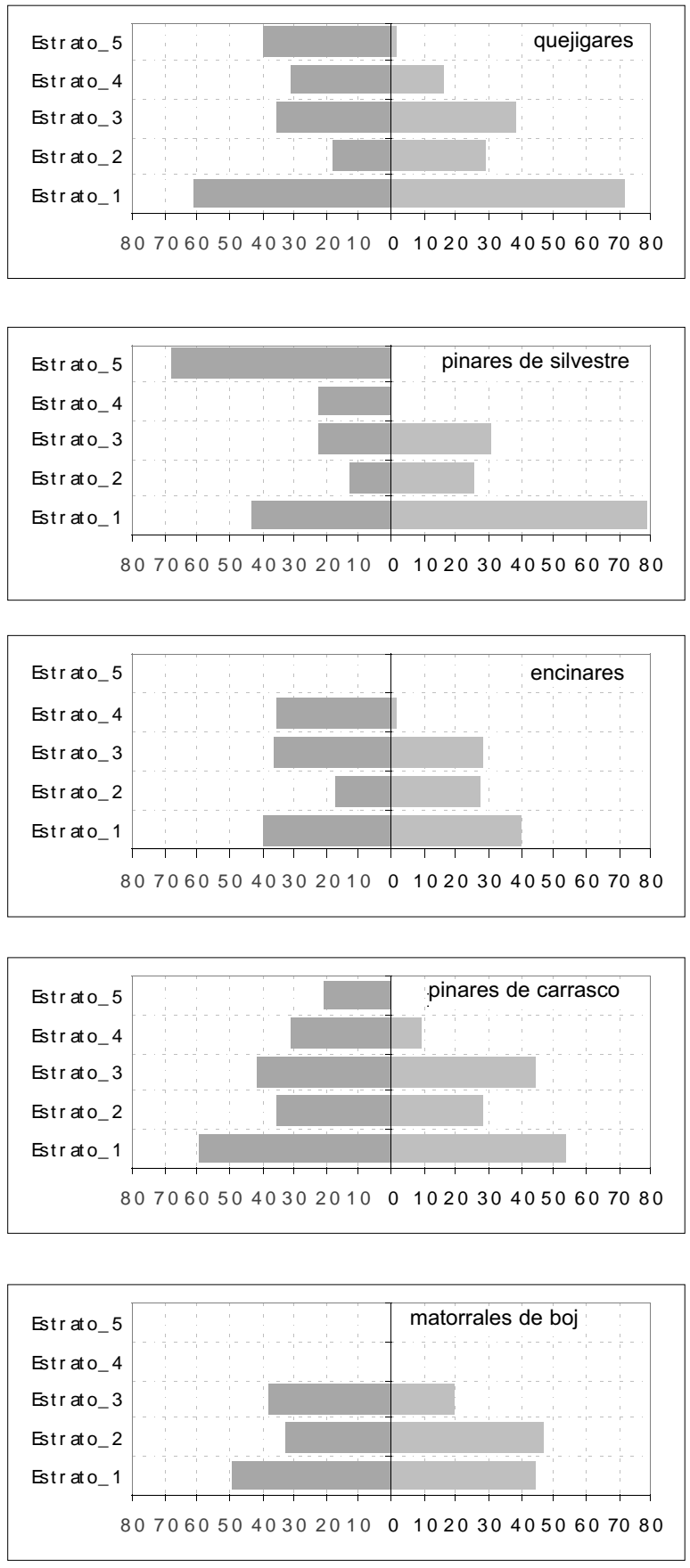
monial del estrato arborescente. En términos generales el recubrimiento vegetal se aproxima al 96,5\%, aunque los valores concretos de los sectores varían notablemente en un espectro comprendido entre $140 \%$ y el $60 \%$.

El índice de variación fisionómica obtenido es ligeramente inferior al de los quejigares $(0.23)$ debido al menor grado de madurez de las formaciones afectadas y a la buena cota de recuperación de los estratos inferiores, arbustivo y arborescente, aunque este último en menor medida ( $4.3 \%$ recuperado). En este caso, a la consabida matorralización de las formaciones arbóreas afectadas por el fuego, le acompaña un menor posicionamiento de individuos -encinas exclusivamente- en los estratos superiores, lo que sin duda también parece estar relacionado con el menor porte de la mayoría de las encinas afectadas.

La composición florística sigue estando presidida por Quercus rotundifoliae, Buxus sempervirens, Genista scorpius y el cortejo de especies pertenecientes a los herbazales de este ámbito fitosociológico. Los estratos arbustivo y subarbustivo están absolutamente dominados por los renuevos y vástagos de Buxus sempervirens, Genista scorpius y Quercus rotundifoliae. Esta última también monopoliza el arborescente debido a que las características morfológicas de sus sistemas radiculares -raíz muy penetrante, axonomorfa y pivotante- aseguraron un abundante número de brotes de raíz y cepa. En cuanto al índice de variación de las relaciones de dominancia, a los buenos niveles de recuperación de la encina y el boj (72.2\% y 83.7\% respectivamente) hay que significar la invasión protagonizada por Genista scorpius con un porcentaje de recuperación del 449.7\% lo que hace que el IVD obtenido sea ligeramente superior al del quejigar (0.23).

Las nuevas formaciones que sustituyen a los pinares de silvestre están constituidas por un desarrollado ESTRATO $1>75 \%$ ), acompañado puntualmente por caméfitos y microfanerófitos (Buxus sempervirens, Rosas sp.) con alturas comprendidas entre los 3 y los $0.5 \mathrm{~m}$. De este modo, mientras que la vegetación de menos de $3 \mathrm{~m}$ ha experimentando incrementos superiores al $130 \%$, los estratos arborescente y el arbóreo no aparecen representados en ninguna de las áreas muestreadas. Esta asimetría tan acusada entre las formaciones originales y las sustitutas $(\mathrm{IVF}=0.31)$ se relaciona, entre otros extremos, con la inexistencia en las formaciones pre-incendio de un sotobosque submediterráneo capaz de recomponerse por brotación con celeridad y, evidentemente, con los problemas de germinación de Pinus sylvestris.

En consecuencia, la escasez de arbustos y la desaparición del efecto inhibidor del pino sobre la germinación de otras especies -aumento de la iluminación, finalización de la emisión de compuestos y toxinas orgánicas-, ha facilitado la fructificación de los bancos de semillas persistentes y del entorno. Estas nuevas condiciones micro-ambientales han precipitado el crecimiento acelerado de una serie de especies -herbáceas perennescapaces de maximizar la obtención de nutrientes tras el incendio (Helictotrichon cantabricum, Brachypodium pinnatum) y de taxones especializados en la colonización de espacios del piso montano como Echinospartum horridum que, al mismo tiempo, inhibe el crecimiento de las plántulas de arbustos y árboles.

Desde el punto de vista de las relaciones de dominancia las variaciones son todavía más acusadas $(I V D=0.31)$. Ninguna de las comunidades analizadas experimenta varia- 
ciones tan intensas como en el caso de los pinares. A ello contribuye especialmente la baja tasa de crecimiento de Pinus sylvestris debido a la ausencia de termodehiscencia de sus frutos y a la escasa viabilidad de sus semillas -escasamente 4 años si no están en lugares adecuados (Tapias y Gil, 2000)-.

El espacio del pino es ocupado ahora por una serie de herbáceas -competidoras tolerantes a restricciones- pertenecientes a los géneros Brachypodium, Bromus, Festuca, Helictotrichon, etc. dotadas de una gran capacidad de propagación vegetativa por medio de rizomas o por macollas; por plantas rastreras, como Fragaria vesca, Hieracium pilosella, asociadas a hábitats pedregosos y rocosos; y por ruderales competitivas y tolerantes a la restricciones como Galium aparine, Ranunculus repens, Primula veris, etc.

Junto a las herbáceas, Buxus sempervirens, Echinospartum horridum y Genista scorpius también ocupan buena parte de este estrato en los ambientes con carácter más montano, aunque es en los estratos arbustivo y subarbustivo, conviviendo con diferentes rosáceas y especies pertenecientes al género Rubus, donde realmente ejercen su dominio. Sólo el boj constituye la mitad de las especies de los estratos 2 y 3 (45\% y el 52\% respectivamente), mientras que la aliaga-Genista scorpius- supone el 12\% del estrato 3 y el $33 \%$ del estrato subarbustivo.

En lo que respecta a los pinares de pino carrasco, las formaciones que los sustituyen muestran un recubrimiento acumulado superior al 135\% y una ocupación media por estrato del $33.8 \%$, lo que supone una disminución de tan sólo 4 puntos en relación con los sectores control (37.5\%) (Tabla 3). Todos los estratos excepto el arbóreo aparecen representados. Sin embargo, destacan especialmente los porcentajes de ocupación del estrato inferior y del arbustivo (53.6\% y $44.3 \%$ respectivamente) respecto a la poca entidad del arborescente y el arbóreo. No obstante, el posicionamiento de individuos en este último nivel, apunta la buena recuperación de estas formaciones $(\mathrm{IVF}=0.20)$

La composición florística de los sectores quemados está presidida por el Pinus halepensis (14,6\%), Genista scorpius (15\%) y Quercus coccifera $(30,8 \%)$, que junto a las herbáceas son las especies dominantes en estos sectores. Entre las especies que han protagonizado las variaciones más acusadas se encuentran Quercus coccifera que duplica su nivel de ocupación y Genista scorpius y el conjunto de herbáceas y graminoides. Aunque Pinus halepensis experimenta un decrecimiento de más del 50\%, mantiene un porcentaje relevante en el conjunto de las especies formadoras de las comunidades de sustitución que se maximiza al representar con exclusividad la vegetación del estrato arborescente, gracias a su portentoso sistema estratégico de adaptación activa al fuego basado en la serotinidad y persistencia de sus piñas termodehiscentes (IVD=0.23). Por su parte, Juniperus sp. debido a la lentitud y dificultad de su germinación apenas aparece en las formaciones que sustituyen estos pinares. Un fenómeno parecido ha sido observado por otros autores (Papió, 1988).

Las formaciones que sustituyen a los matorrales de boj se estructuran en torno al dominio de los ESTRATOS $_{1 / 2}$ (44.8 y 47.1\% respectivamente), acompañados en la mayoría de los casos por un estrato de carácter arbustivo con presencia en torno al $20 \%$. En 
términos generales, los niveles de recubrimiento acumulado se sitúan en torno al $100 \%$ alcanzando la superficie descubierta valores próximos al $26 \%$, lo que supone casi el tripe de la registrada en los sectores control. La importancia relativa de Buxus sempervirens es muy similar en los tres estratos que conforman las comunidades que sustituyen a los bojedales quemados. Estos últimos, como se ha visto más arriba presentan grandes diferencias; la abundancia de las especies es una función negativa de la talla que presente.

\section{3 Índices de variación y análisis cluster}

En atención al índice de variación en las relaciones de dominancia se han detectado diferencias estadísticamente significativas entre cualquiera de las comunidades en relación con los pinares de silvestre $(P=0.0025)$ (Figura 3). En cuanto al índice de variación fisionómica, la mayor madurez y estructuración de los pinares de silvestre y de los quejigares contrastan ostensiblemente con el resto de comunidades, en especial con los encinares que son los que menos diferencias computan $(P=0.0056)$ (Figura 4$)$.

Figura 3. Intervalos de confianza para los valores medios del indice de Variación de las Relaciones de Dominancia (IVD) por formación vegetal

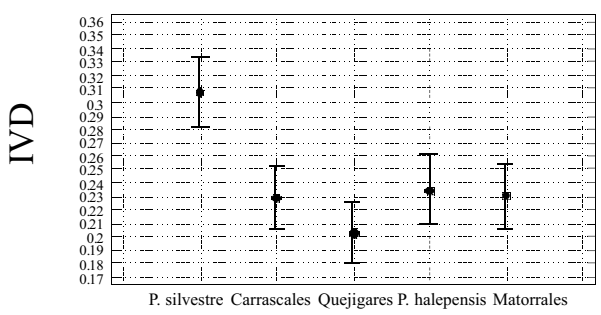

Figura 4. Intervalos de confianza para los valores medios del indice de Variación Fisionómica (IVF) por formación vegetal

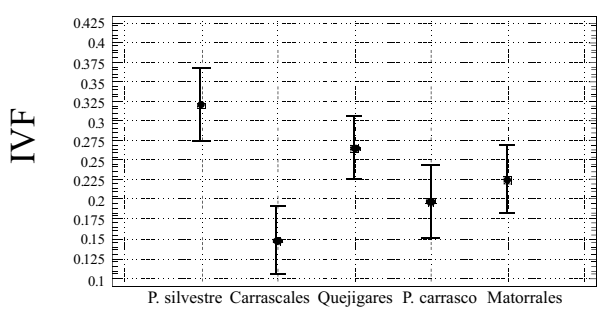

Figura 5. Dendrograma del análisis cluster Ward's Method,Squared Euclidean

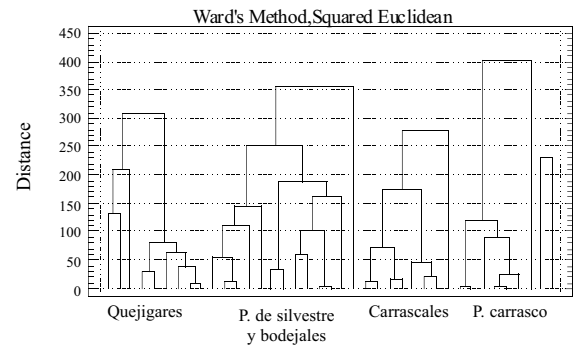


El análisis cluster aplicado confirma la correspondencia entre las formaciones que colonizan las zonas quemadas y sus antecedentes excepto en el caso de los pinares de silvestre. En este sentido, atendiendo a la composición florística y a la ordenación por estratos, se han identificado cuatro tipos principales y un quinto más específico tan sólo integrado por dos observaciones.

El primer conglomerado constituye el $23.08 \%$ de las observaciones (Figura 5), e integra 8 de las nueve áreas test realizadas en los quejigares incendiados y una novena correspondiente a un pinar de silvestre perteneciente al incendio de la Umbría de Aineto. Por lo tanto, este primer cluster sintetiza las características de las formaciones que sustituyen al quejigar prepirenaico descritas anteriormente.

El segundo cluster constituye el $33.33 \%$ de la muestra (13 observaciones) y presenta, en relación con la estructura inicial, una composición mucho más heterogénea que el primero. En este caso se trata de 6 observaciones correspondientes a pinares de silvestre, otras 6 pertenecientes a matorrales de boj y erizón, y una de quejigar. El rasgo más diferenciador de este conglomerado con el resto reside en la importancia espacial que presentan Genista scorpius y Buxus sempervirens, aunque sólo en el caso de la primera especie se han encontrado diferencias estadísticamente significativas, y tan sólo con el primer conglomerado. La interpretación del perfil de este conglomerado trasluce la escasa capacidad de recomposición de los pinares de silvestre analizados y la reducida diferenciación con la respuesta de comunidades mucho más degradadas, los matorrales de boj.

En términos generales, con el paso del fuego los pinares albares presentan una sintomatología que los conecta directamente a etapas, comparativamente, mucho más recientes en el proceso natural de progresión hacia fases maduras. Así se desprende de las importantes diferencias florístico-fisionómicas que se han computado como consecuencia de la combustión de la cubierta vegetal. Catorce años después del fuego, las estrategias anatomo-fisiológicas de las especies principales que los integran y las propias características estructurales de los paisajes donde se desarrollan no han sido capaces de impulsar a estos sistemas a estadios próximos a los de la vegetación preexistente. Por tanto, el paso del fuego ha supuesto en estas comunidades arbóreas una importante ruptura en el proceso de sucesión ecológica que funcionaba previamente.

En el tercer y cuarto cluster volvemos a comprobar la correspondencia entre conglomerados. El tercero recoge el $20.51 \%$ de las observaciones, todas ellas incluidas en los carrascales submediterráneos, siendo Quercus rotundifoliae diferenciador del resto de los conglomerados -diferencias estadísticamente significativas con los cuatro restantes. El cuarto cluster recoge el $17.95 \%$ de las observaciones en este caso todas ellas incluidas en los pinares de carrasco. Finalmente el último conglomerado integra tan sólo el 5.13\% de la muestra, dos miembros, que se corresponden con respuesta específica de los matorrales. Se trata de dos matorrales de componente mediterránea caracterizados por el predomino de pinus nigra, rosas, etc. 


\section{Conclusiones}

Los resultados obtenidos en este estudio coinciden con los patrones de comportamiento postincendio descritos por otros autores para comunidades mediterráneas (Trabaud, 2002). Las diferencias entre comunidades dependen de las diferentes formas de restablecimiento de las especies que lo integran. Se puede concluir reseñando tres aspectos principales.

En primer lugar, el descenso generalizado en los niveles de recubrimiento vegetal porcentajes acumulados por estratos- y el desarrollo desproporcionado de los estratos inferiores, muestras evidentes del todavía inconcluso proceso de reconstrucción y del menor grado de evolución de las formaciones postincendio.

En segundo lugar, la gran similitud, en términos generales, de la composición florística de los espacios contrastados, lo que guarda una estrecha relación con la elevada resiliencia de las comunidades afectadas. Sin embargo, en algunos estratos se advierte cierto enriquecimiento florístico e importantes variaciones en el reparto de las especies principales, que se asocia a las nuevas relaciones de competencia que se establecen por el espacio, como consecuencia de la transformación micro-ambiental del paso del fuego (luminosidad-nutrientes-actividad erosiva).

En tercer lugar, se constata la existencia de diferencias en la respuesta de las comunidades forestales analizadas. En este sentido, los bosques subesclerófilos dominados por Quercus rotundifoliae y Quercus gr. cerrioides presentan una mayor capacidad de cicatrización que los dominados por coníferas, especialmente si se trata de comunidades de sustitución o instaladas de modo artificial. Por otra parte, las comunidades más degradadas, aquéllas que representan etapas tempranas en el contexto de un marco climácico -formaciones arbustivas y subarbustivas submediterráneas presididas por Buxus sempervirens o Quercus coccifera- detentan una mayor facilidad para su reinstalación y reconstrucción tras el fuego como consecuencia de la menor cantidad de energía que demandan; sin embargo, las comunidades resultantes manifiestan los signos de degradación edafo-vegetal más acusados. No obstante, tan sólo se han detectado diferencias estadísticamente significativas cuando interviene la unidad pinares de silvestre. Por tanto, se constata la distinta naturaleza de la respuesta de los sistemas dominados por el pino silvestre y el resto de unidades submediterráneas y mediterráneas dominadas por especies muy resilientes.

\section{Bibliografía}

Badía, D., Marti, C., Royes, E. y Galindo, S. (1995). Influencia del fuego en la sucesión vegetal de un coscojar en el noreste de España. Historia Natural, 93: 83-93.

Bertrand G. (1966). Pour une etude géografphie de la végétation. Rev. Geogr. Pyr. Sud. Ouest., 37: 129-243.

Braun-Blanquet, J. (1979). Fitosociología. Bases para el estudio de las comunidades vegetales. Blume, Madrid. $820 \mathrm{pp}$.

Braun-Blanquet, J. y De Bolós, O. (1957). Les groupements vegetaux du Bassin de l'Ebre et leur dinamisme. Ann. Est. Exp. Aula Dei, 5 (1-4), 266 pp. 
Gordon, M. y Poissonet, J. (1973). Quatre thémes complémentaires pour la cartographie de la végétation et du milieu. Bull. Soc. Lang. de Geograpie, 6:329-356.

HANeS, T.J. (1971). Succession after fire in the chaparral of southern California. Ecol. Monogr., 41: 27-52.

Jarvis, P.G. y Jarvis, M.S. (1964). Growth rates of woody plants. Physiologia Pl. 17: 654-666.

MAY, T. (1991). Observaciones y reflexiones sobre el comportamiento tras fuego de algunas especies de la zona mediterráneas de Andalucía Oriental. Ecología, 5: 125-134.

NAVEH, Z. (1990). Fire in the Mediterranean - A landscape ecological perspective. En: Fire in ecosystem dynamics (J. G. Goldammer \& M. J. Jenkins, Ed.), SPB Academic Publishing, 1-20, The Haghe.

PAPIÓ, C. (1988). Respuesta al fuego de las principales especies de la vegetación de Garraf (Barcelona). Orsis, 3:87-103.

PAPIÓ, C. (1994). Ecología del foc i regeneració en garrigues i pinedes mediterrànies. Insitut d'Estudis Catalans. ASC VIII, 392 pp., Barcelona.

Pérez CABello, F. (2002). Paisajes forestales y fuego en el Prepirineo oscense: un modelo regional de reconstrucción ambiental. Serie Investigación, 33. Consejo de Protección de la Naturaleza en Aragón, D.G.A., 360 pp., Zaragoza.

TAPIAS, R. y GIL, L. (2000). Adaptación reproductiva de las especies forestales ante el fuego, en Vélez, R. (coord.): La defensa contra incendios forestales. Fundamentos y experiencias. McGraw-Hill, pp. 4.36-4.66, Madrid.

TÁrrega, R., CAlvo, L. y Luis-CAlABuig, E. (1990). Estudio comparativo de la composición florística en la regeneración post-fuego de ecosistemas de Quercus pyrenaica Willd.. Acta Botánica Malacitana, 15: 331-339.

TÁrrega, R. y Luis-CAlabuig, E. (1987). Effects of fire on structure, dynamics and regeneration of Quercus pyrenaica ecosystems. Ecologia Mediterranea, 13 (4): 7986.

TÁrrega, R. y Luis-CAlabuig, E. (1989). Análisis temporal durante tres años consecutivos de las primeras fases de regeneración post-fuego en robledales de la provincia de León. Studia Ecologica, 6: 205-216.

Trabaud, L. (1990). Fire resistance of Quercus coccifera L. Garrigue. En: Proceedings of the Third International Symposium on Fire Ecology (J. G., Goldammer \& M.J. Jenkins, ed.). SPB Academic Publishing. 21-32, The Haghe.

Trabaud, L. y ValinA, J. (1998). Importance of tree size in Pinus halepensis fire survival. En: Fire Management and Landscape Ecology (L. Trabaud ed.). International Associatión of Wildland Fire. 189-196.

Trabaud, L. (2002). Post-fire vegetation recovery and dynamics in the Mediterranean area, En: Fire, Landscape and biodiversity: An appraisal of the effects and effectiveness. Lectures from de 3rd International Summer School on the Environmet (Pardini, G. y Pintó, J. eds.). Diversitas [29]. Universitat de Girona. Institud de Medi Ambient. 39-56, Girona.

Vera DE LA FUENTE, M.L. (1994). Regeneración de un aulagar con Ulex europaeus después de un incendio en el norte de España. Pirineos, 143-144: 87-98. 